\title{
Multifractality and singularity in average temperature and dew point across India
}

\author{
Rajdeep Ray ${ }^{*}$, Siddhartha Dey ${ }^{2}$, Mofazzal Hossain Khondekar ${ }^{3}$ and Koushik Ghosh ${ }^{4}$ \\ Assistant Professor, Department of Electronics and Communication Engineering, Dr. B. C. Roy Engineering \\ College, Durgapur, India ${ }^{1}$ \\ M. Tech Scholar, Department of Electronics and Communication Engineering, Dr. B. C. Roy Engineering College, \\ Durgapur, India ${ }^{2}$ \\ Professor, Department of Applied Electronics and Instrumentation Engineering, Dr. B. C. Roy Engineering College, \\ Durgapur, India ${ }^{3}$ \\ Assistant Professor, Department of Mathematics, University Institute of Technology, University of Burdwan, \\ Burdwan, India ${ }^{4}$
}

\section{(C2018 ACCENTS}

\begin{abstract}
In the present work we have taken into account the observations of daily temperature and dew point of seven different stations viz. Kolkata, Ahmedabad, Bhopal, Agartala, Mumbai, Chennai and New Delhi. Here the spatial average of temperature and dew point of these seven stations are the signals under observation. A thorough analysis has been made to examine the multifractality and singularity within the signals. The multifractal detrended fluctuation analysis (MFDFA) and wavelet transform modulus maxima (WTMM) based on continuous wavelet transform method are being exploited to serve the present purpose. The results reveal multifractal nature of the present signals.
\end{abstract}

\section{Keywords}

Indian climate, Time series analysis, Multifractal detrended fluctuation analysis, Wavelet transform modulus maxima (WTMM), Continuous wavelet transform (CWT), Wavelet shrinkage, Hölder exponent, Hausdorff dimension, Singularity spectrum.

\section{Introduction}

The issue of variation in weather dynamics subject to increase in average temperature of earth's atmosphere, resulting in change of climate has become a field of exquisite interest during last few decades. Change in weather dynamics affects numerous important areas and blooming domain, including forestry, agriculture, coastal zones and energy. Furthermore, the financial and social acquirement is threatened failing to assure the safety of the environment. All studies in this regard states increase in mean annual temperature and dew point in India. This global warming has generally been followed by increase in diurnality, except in winter, pre-monsoon and post-monsoon seasons of northern India [1]. Srivastava et al. [2] monitored increasing trends of annual means, maxima and minima for both temperature and dew point around south of $23{ }^{\circ} N$ and cooling trends for the same around north of $23^{\circ} \mathrm{N}$.

*Author for correspondence

107
Some of the other researchers [3-6] have come to the conclusion from their research that large part of Indian subcontinent can firmly view a trend which is increasing in mean the temperature and dew point in winter and post-monsoon seasons. Increasing trends in temperature and dew point can be visible over major southern and central parts of India in all seasons and the northern part of India in the postmonsoon season. The diurnal temperature and dew point range increase most considerably in the monsoon season mainly because of the rise in daytime temperature and dew points. Night time temperature and dew point remain fairly constant except over northern India where it may fall slightly. Time series analysis of mean annual temperature and dew point and distinctive criteria representing seasonal fluctuations of temperature and dew point for different Indian cities was performed by Jagannathan and Parthasarathy [7]. The mean annual temperature and dew point shows an increasing trend in Allahabad, Bangalore, Kolkata, and Mumbai and a decreasing trend at Fort Cochin [8]. These multipurpose studies essentially state that the 
temperature and dew point has a diverse spatial as well as temporal trend of fluctuation. It is normally tough to obtain complete dynamics of the temperature and dew point, without noticing to the statistical attribute of this dynamics due to the stochasticity and complexity of the variations in the temperature and dew point. This in turn suggests us to apply suitable stochastic investigation to explore the growth and substantial properties of the temperature and dew point produced by such fluctuations. Some trends and non-stationarity may also affect the original fluctuations as there are some drawbacks in the experimental preparation for calibrating the fluctuations and the suitability for obtaining data in some instances. Hence, in order to surmise expensive statistical characteristics of the original fluctuations and avert fraudulent disclosure of correlations, a vigorous technique which must be unresponsive to any trend must be used. There should have the same probability distribution function for both correlated and uncorrelated time series which are may be monofractal or multifractal in type. Only one scaling exponent is used to describe monofractal signals. The simple monofractal scaling behavior cannot be seen in many time series. Some of the instances have a cross over (time) scales dividing the system with different scaling exponents. In some other instances, there exist a more complex scaling response, and different component of the series need different scaling exponents. Even in more complex instances, multiple intertwine fractal subsets of the time series exhibit such different scaling behaviour. For these instances a multitude of scaling exponents is needed for a full representation of the scaling behaviour, and a multifractal analysis must be done [9]. In our surroundings, two distinct kind of multifractality in time series can be differentiated: (i) Multifractality for fluctuations caused by a broad probability density function (PDF). In multifractality for fluctuations scenario, the multifractality cannot be eliminated by rearranging the series. (ii) Multifractality as a result of different (time) correlations for small and large fluctuations. In this case the rearranging series will show weaker multifractality than the original series, if both these two types of multifractality are present [10]. In order to examine the scaling structure or fractality of the average temperature and dew point in India we will exploit multifractal detrended fluctuation analysis (MFDFA) [11], continuous wavelet-based methodologies such as continuous wavelet transform (CWT) and the wavelet transform modulus maxima (WTMM) [12] which, as revealed, propose key edge as contrast to the more conventional multifractal point of views such as box counting and structure function techniques. They are immune to nonstationarity as well as both the processes efficiently eliminate the underlying polynomial trends and emphasize on the analysis of the fluctuation. In this work the daily mean temperature and dew point of seven different stations (Kolkata $\left(22.57{ }^{0} N\right.$ ), Ahmedabad (23.04 $\left.{ }^{0} N\right)$, Bhopal $\left(23.26{ }^{0} N \quad\right.$ ), Agartala (23.83 $N$ ), Mumbai (19.02 $N$ ), Chennai 0

(13.06 $N$ ) and New Delhi (28.34 $N$ )) from 9th October 1996 to 1st February 2013 observed by National Oceanic and Atmospheric Administration (NOAA) [13] have been considered. These seven stations represent different geographical domains in India. It is well expected that a thorough study over these stations can dispense a good understanding of the dynamics of Indian weather as a whole. The signal variables like scaling exponents, multifractal scaling exponents and generalised multifractal dimensions have been computed to quantify the multifractality of the signal. To track the singularities in the signal, the singularity strength or Hölder exponent have been computed and the Hausdorff dimension or singularity spectrum has been also calculated. Multifractal analysis reveals the existence of a very sporadic multifractal structure (a wide spectrum of singularities) in the present nonstationary temperature and dew point time series.

\section{Methodology}

2.1Multifractal analysis using MFDFA (Multifractal detrended fluctuation analysis) The MFDFA is a complete approach for establishing scaling response in a signal in existence of possible trends without intending their origin and shape [14]. Its advantage over the traditional techniques (e.g. the power spectrum) as it allows the identification of inherent active features, like scaling and long-range correlations, established in an apparently nonstationary time series, hence averting the fraudulent detection of possible scaling, which may be an artefact of external trends [15]. In case of nonstationary signals there exists an intrinsic fluctuation as well as the polynomial trend within the signal. To obtain the satisfactory scaling property of such signals it is necessary to firmly differentiate between them. In these types of applications MFDFA can be used. The polynomial trends can be removed but the sinusoidal trends cannot be eliminated by MFDFA. These sinusoidal trends can be removed using SVD (singular value decomposition) method before 
applying MFDFA [16].

Considering a trend-free time-series signal $t(i)$ as the increment of a random walk process about the average value $\langle t\rangle$, the 'trajectory '(or 'profile') $U(j)$ of the time series is obtained from the relation as given below

$$
U(j)=\sum_{i=1}^{j}[t(i)-\langle t\rangle] \text { for } i=1, \ldots, N
$$

The profile $U(j)$ is then divided by $N_{s}$ nonoverlapping components of equivalent length $s$. The time series of length $N$ may not necessarily be an integral multiple of the time scale $s$; thus a small portion of the trajectory can exist at the termination part of the trajectory. In order to achieve a greater degree of efficiency in estimation process, the identical procedure (forward direction procedure) is restated from other end (backward direction procedure) as well. Hence, $2 N_{s}$ segments can be achieved by combining these two.

The local trend for every $2 N_{s}$ segment is then computed by a least-square fit of the series. Then the variance for forward direction procedure is determined as

$$
F^{2}(s, v)=\frac{1}{s} \sum_{j=1}^{s}\left[U\{(v-1) s+j\}-U_{v}(j)\right]^{2}
$$

for every segment $v, v=1, \ldots, N_{s}$. For backward direction procedure

$F^{2}(s, v) \equiv \frac{1}{s} \sum_{j=1}^{s}\left[U\left\{N-\left(v-N_{s}\right) s+j\right\}-U_{v}(j)\right]^{2}$

For $v=N_{s}+1, \ldots, 2 N_{s}$. Here $U_{v}(j)$ is known as fitting polynomial in $v$ segment.

After detrending the time series, the $q$ th-order fluctuation function $F_{q}(s)$ can be obtained by performing an average $F^{2}(s, v)$ over the whole segments $\quad v$ and it is given by $F_{q}(s) \equiv\left[\frac{1}{2 N_{s}} \sum_{v=1}^{2 N_{s}}\left\{F^{2}(s, v)\right\}^{q / 2}\right]^{1 / q}$

and the moment $q$ is of any real value other than zero.

However, the direct averaging process as stated in (4) totally fails to calculate the fluctuation function $F_{q}(s)$ for $q=0$ because of the diverging type of the exponent [12]. Hence, a logarithmic averaging process can be applied to calculate the fluctuation function $F_{q}(s)$ for (i.e. $\left.F_{0}(s)\right)$ by using the following equation,

$$
F_{0}(s) \equiv \exp \left[\frac{1}{2 N_{s}} \sum_{v=1}^{2 N_{s}} \ln \left|F^{2}(s, v)\right|\right]
$$

It is evident that the value of $F_{q}(s)$ increases when $s$ increases. Certainly, $F_{q}(s)$ is based upon the DFA of order $m . F_{q}(s)$ is constructed which is described for $s \geq m+2$.

Now the scaling behaviour of the fluctuation functions can be established by analyzing the plots of $\log F_{q}(s)$ versus $\log \mathrm{s}$ for every values of $q$. Whenever the series $x(i)$ are long-range power-law correlated then $F_{q}(s)$ increases with crease of $s$, as a power-law is given by

$$
F_{q}(s) \sim s^{h(q)}
$$

Generally the exponent $h(q)$ is relying on. In case of stationary time series, $h(2)$ is similar to the wellknown Hurst exponent $H$. Therefore, the function $h(q)$ will be called as generalized Hurst Exponent. When the values of $q$ are considered to be positive, then the segments having large variance $F_{s}^{2}(v)$ (i.e. large deviations from the equivalent fit) will influence the average $F_{q}(s)$. So for positive values of $q, h(q)$ states the detailed scaling behaviour of the segments with large fluctuations. Normally the multifractal series having smaller scaling exponent $h(q)$ is characterized for large fluctuations. Furthermore for negative values of $\mathrm{q}$, the segments $v$ with small variance $F_{s}^{2}(v)$ will influence the average $F_{q}(s)$. So in case of negative values of $q$, $h(q)$ defines the scaling behaviour of the segments with small fluctuations, which are described by a larger scaling exponent. The relation of $h(q)$ and the multifractal scaling exponents $\tau(q)$ is shown as follows [17]

$\tau(q)=q h(q)-1$ 
2.1.1Determination of the order of trend to detrend the signal in MFDFA

Since the time series detrending is done by the subtracting the polynomial fits from the profile as shown in equation (3), DFA of different orders varies in their ability of removing trends in the series. The sequence of this polynomial decides the order of the trend in the time series such as linear, quadratic, cubic, or higher order trends which are to be eliminated and the fitting procedures are conventionally called MFDFA1, MFDFA2, and MFDFA3 and so on. MFDFAm eliminates the $(m-1)^{\text {th }}$ order trend in the time series or $m^{\text {th }}$ order trends in the profile [14]. Hence by comparing the outcomes for dissimilar orders of DFA the polynomial trend type can be calculated easily in the time series.

\subsection{Wavelet transform modulus maxima (WTMM)} based on continuous wavelet

WTMM [18-20] is a procedure for identifying the fractal dimension of a signal. Moreover WTMM has the capability of partitioning the time and scale domain of a signal into fractal dimension regions. This is achieved due to its potentiality of inspecting the multi-scale dimensional characteristics of a signal. Possibly it also informs about the sources of these characteristics. WTMM technique uses continuous wavelet transform (CWT) to discover singularities in the signal.

2.2.1Choice of suitable mother wavelet for continuous wavelet transform (CWT)

Wavelet transform disbalances signal energy under consideration. Lowest balance implies lowest energy and in turn maximum stability. The entropy of the most stable transform will be the least and the best one. The minimum entropy will be the transform which produces largest ratio of few large coefficients to many small coefficients. In order to select the best possible mother wavelet for a particular signal we have chosen the method which is based on decreasing the entropy of the wavelet transformed matrix [21, 22].

$$
E(W)=-\sum_{i, j} c_{i, j}^{\prime} \log c_{i^{\prime} j}^{\prime}
$$

Where $c_{i, j}^{\prime}$ are the nonnegative normalized wavelet coefficients, that is, $c_{i, j}^{\prime}=\left|c_{i, j}\right| / \sum\left|c_{i, j}\right|$. The best WT will minimize $E(W)$ from the repository of possible mother wavelets.

2.2.1.1Wavelet shrinkage

In the process of disbalancing the energy of the signal, the wavelet transform produces some insignificant information. To remove those insignificant information the coefficients of are to be shrinked to zero or shrinked toward certain coefficients.

Normally, there are two categories of thresholding present viz. hard and soft. Hard thresholding can be written as

$c_{i, j}= \begin{cases}0, & \left|c_{i, j}\right|<\lambda \\ c_{i, j}, & \left|c_{i, j}\right| \geq \lambda\end{cases}$

Where all $\left|c_{i, j}\right|<\lambda$ are set to zero and all other are kept for consideration.

Soft thresholding may be written as

$$
c_{i, j}=\operatorname{sign}\left(c_{i, j}\right)\left(\left|c_{i, j}\right|-\lambda\right)
$$

where all $\left|c_{i, j}\right|<\lambda$ are consider to be zero and others are shrunk towards zero by a threshold amount of $\lambda$. In this work a universal threshold has been used to obtain the threshold value $\lambda$. The universal threshold employs universal sequence given by

$$
\lambda_{n}^{u}=(2 \log n)^{1 / 2}
$$

\subsection{Continuous wavelet transform (CWT)}

The coefficients of CWT, $W f(z, d)$ are given by

$$
W f(z, d)=\frac{1}{z} \int_{-\infty}^{\infty} f(y) \psi\left(\frac{y-d}{z}\right) d y
$$

Where $z, \mathrm{~d}$ are real, $z>0$ for CWT.

The mother wavelet is stretched or compressed to the required resolution by scale parameter $z$ whereas the analyzing wavelet is shifted to the desired location by the translation parameter $d$. Usually, the mother wavelet $\psi(x)$ alone have to be of zero mean and orthogonal to some lower order polynomials up to degree $p$ so as

$\int y^{q} \psi(y) d y=0, \quad \forall q, \quad 0 \leq q \leq p$

By conserving both scale and location (time, space) information and filtering out the trends, the continuous wavelet transform reveals the local characteristics and mapping of the variation of nonstationary signals, and more precisely their singularities. Thus from (12), the wavelet transform 
of $f(y)$ can be represented as

$$
\begin{aligned}
W f(z, d) & =\frac{1}{z} \int_{-\infty}^{\infty} f(y) \psi^{(p)}\left(\frac{y-d}{z}\right) d . \\
& =s^{p} \frac{d^{p}}{d x^{p}} W f(z, d)
\end{aligned}
$$

Equation (14) depicts that if the derivatives are of higher order then greater vanishing moments, i.e., the local polynomial trends of higher order would be removed.

2.4Quantification and analysis of wavelet transform modulus maxima (WTMM)

Let $\left[V_{n}(s)\right]$, where integer is the position (time) of all local maxima at a finite scale $s$ is taken. After calculating the summation of the $q$ 's power of all wavelet transform modulus maxima (WTMM), the partition function is obtained as follows:

$Y(q, s)=\sum_{n}\left|W f\left(s, V_{n}\right)\right|^{q}$

Where is of real value except zero.

From the deep analogy of the multifractal formalism to thermodynamics [23, 19] the scaling exponent $\tau(q)$ is related to the partition function as follows

$Z(q, s) \sim s^{\tau(q)}$

The plots of $f(\alpha)-\alpha$ (where $\alpha$ is known as Lipschitz-Hölder exponent) and $D(q)$ can be estimated from the $\tau(q)$ characteristics. Exploiting Legendre transform the relation between $\tau(q)$ and singularity spectrum $f(\alpha)$ using (17) and (18).

$$
\begin{aligned}
& \alpha(q)=\frac{d \tau(q)}{d q} \\
& f(\alpha)=q \alpha-\tau(q)
\end{aligned}
$$

The generalized multifractal dimension $D(q)$ is related to $\tau(q)$ as

$$
D(q)=\frac{\tau(q)}{q-1}, \quad q \neq 1
$$

Here the moment is taken from to with the step size increase of 0.25 and the scale from 2 to 1024 with the step size increase of 2 .

\section{Results and discussion}

$h(q)$ for different values of $q$ can be estimated by utilizing the linear regression to the plots of $\log$ $F_{q}(s)$ versus $\log s$, as in (6). Here, the moment $q$ is taken from -10 to +10 with the step size increase of 0.25 , the scale ranges from 30 to 118 $(\leq N / 5)$ with the step size increase of 1 for temperature and from 15 to 170 with the increment of 2 for dew point signal [11].

Figure 1 shows the comparison of the plots of $\log$ $F_{q}(s)$ versus $\log s$ for different values of $q$ to the average temperature data using MFDFA1, MFDFA2, MFDFA3 and MFDFA4. Whereas Figure 2 shows the comparison of the plots of $\log F_{q}(s)$ versus $\log$ $s$ for different values of $q$ to the average dew point data using MFDFA1, MFDFA2, MFDFA3 and MFDFA4.
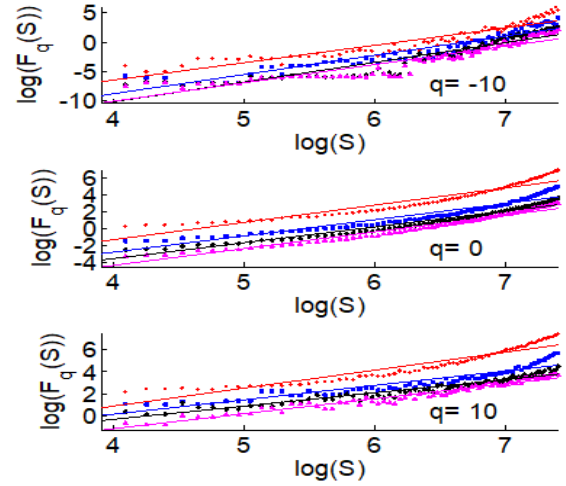
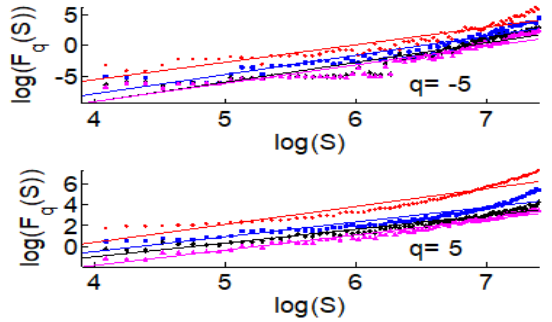

- MF-DFA1

- MF-DFA2

- MF-DFA3

- MF-DFA4

Figure 1 Log versus log plots for MFDFA1, MFDFA2, MFDFA3 and MFDFA4 for average temperature signal Figure 1 shows that the difference between the slopes 
Ray et al.

of the plots of $\log F_{q}(s)$ versus $\log s$ for MFDFA1, MFDFA2, MFDFA3, and MFDFA4 which are of very small for all considered values of $q$. On the other hand from Figure 2 it is evident that in case of dew point the difference between the slopes of the plots of $\log F_{q}(s)$ versus $\log s$ for MFDFA1, MFDFA2 and MFDFA3 are of very small for all values of $q$ and for MFDFA4 differs for all values of $q$. So the time series for average temperature signal may have trend up to fourth-order, whereas average dew point signal may have trend up to third-order, and hence, MFDFA4, MFDFA3 are the respective suitable choices to remove trends from the corresponding signals.

We have used the repository of mother wavelets which is based on increasing coefficients of Daubechies, Coiflet and Symmlet wavelets along with the Haar and Gauss3 wavelet [24].
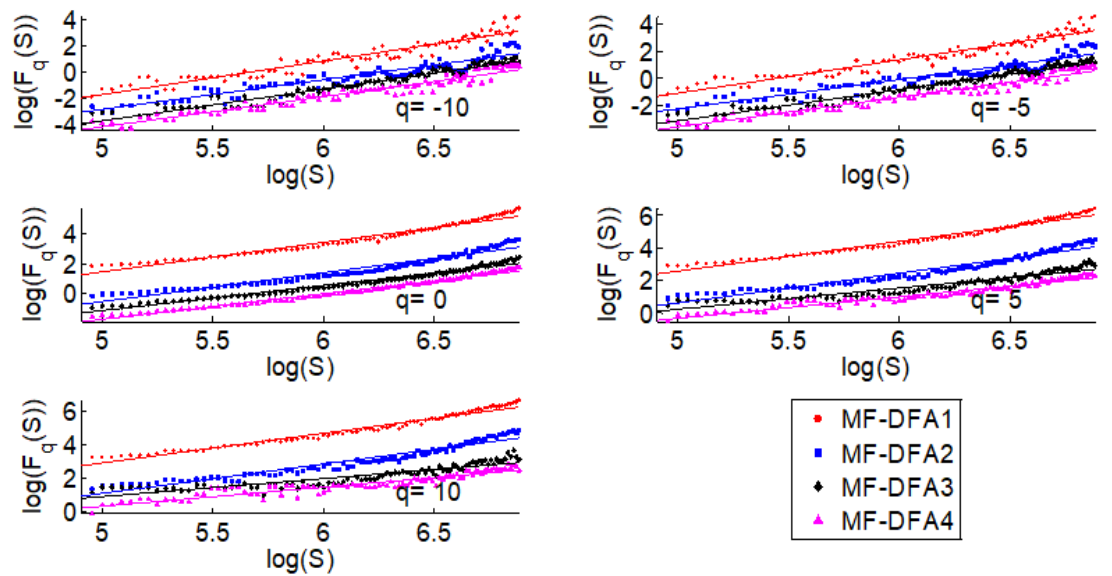

Figure $1 \log F_{q}(s)$ versus $\log s$ plots for MFDFA1, MFDFA2, MFDFA3 and MFDFA4 for average dew point signal

Figure 3(a) and Figure 3(b) are plots of entropy scores for the different mother wavelets. Plots clearly show that the entropy score is minimum for Db8 (Daubechies wavelet of order 8) in Figure 4 represents the variation in the plots of $\log F_{q}(s)$ versus $\log s$ with MFDFA4 for temperature (Figure. $4(a))$ and with MFDFA3 for dew point signal (Figure. $4(b)$ ) for $q=-10$ to +10 with a step size of 4. The mean $F_{q}(s)$ for all the scales $s$ is represented by $F(q)$. Figure 5 and Figure 6 depicts the relative study of the plots of $F(q)$ against $q$, $\tau(q)$ against $q, D(q)$ against $\quad q, \alpha(q)$ against $q$, $f(\alpha)$ against $\alpha, f(\alpha)$ against $\alpha$ and $f(\alpha)$ against $q$ for temperature and dew point data signals respectively. Extrapolating the curves in Figure 5(f) and Figure $6(f)$, we have obtained the results for $\alpha_{\text {min }}(f=0), \alpha_{\text {max }}(f=0)$ and $\alpha\left(f=f_{\text {max }}(\alpha)\right)=\alpha_{0}$ which are tabulated in Table 1

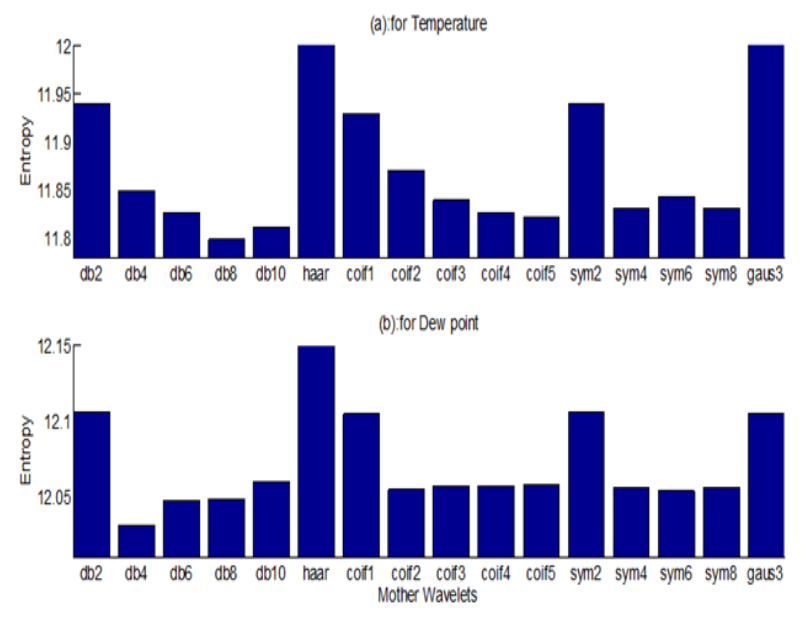

Figure 3 Plots of entropy scores for the different mother wavelets

Figure 7 shows $\log Z(q, s)$ against $\log s$ using WTMM for temperature (Figure 7(a)) and for with MFDFA3 for dew point signal (Figure $7(b)$ ) for 
$q=-10$ to +10 with a step size of 4 . The mean $Z(q, s)$ taken for all the scales $s$ is represented by $Z(q)$.

Figure 8 depicts the comparison of the plots of $Z(q)$ against $q, h(q)$ against $q, \tau(q)$ against $q, \quad D(q)$ against $q, \alpha(q)$ against $q, f(\alpha)$ against $\alpha$ and $f(\alpha)$ against $q$ for temperature and dew point data signals respectively.

The results for $\alpha_{\text {min }}(f=0), \quad \alpha_{\text {max }}(f=0)$ and $\alpha\left(f=f_{\max }(\alpha)\right)=\alpha_{0}$ can be obtained extrapolating the curves as in Figure $8(f)$ which are tabulated in Table 1 .

\section{(a): For Temperature}

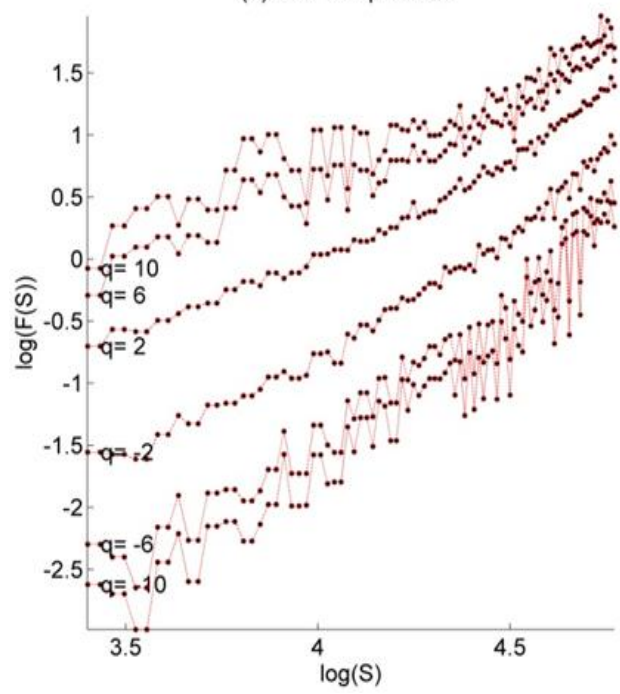

(b): For Dew point

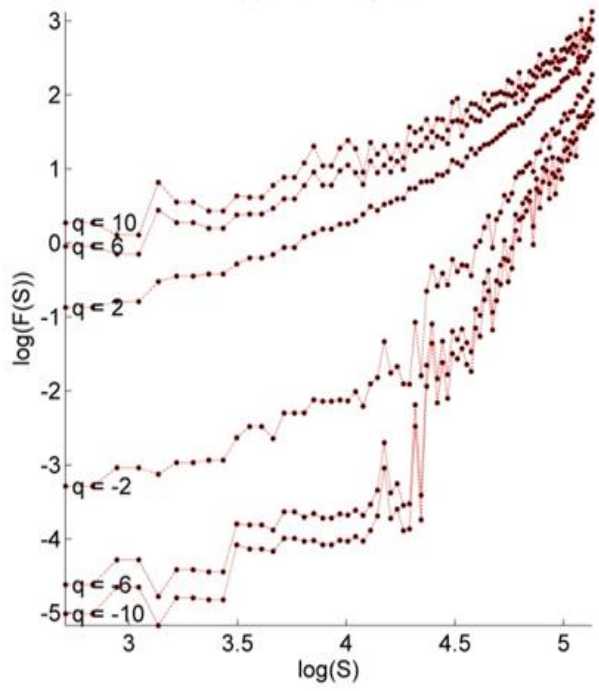

Figure $2 \log F_{q}(s)$ versus $\log s$ plots for different $q$ using MFDFA: (a) For Temperature, (b) For Dew point MFDFA for Original,Surrogated and Shuffled signal for Temperature Signal
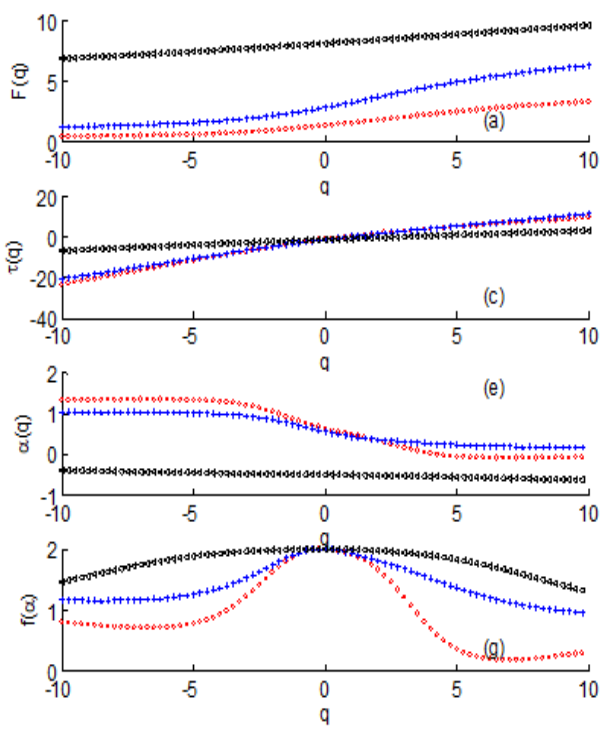

(b)
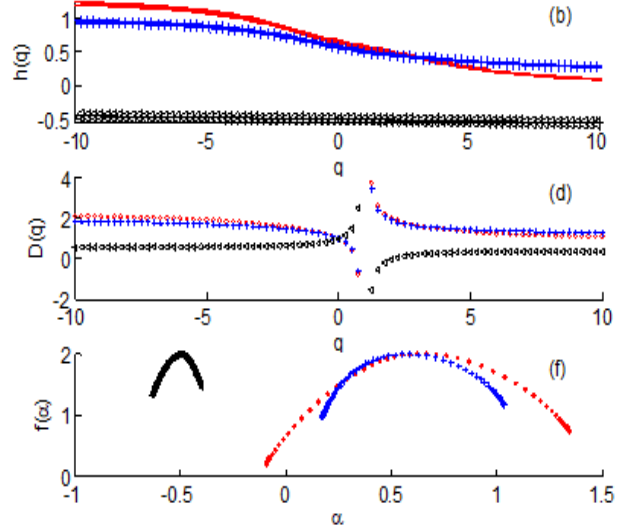

- Original

+ Surrogate
4 Shuffle

Figure 5 Comparison between original, surrogated and shuffled for Temperature Data using MFDFA4: (a) $F(q)$, (b) $h(q),(\mathbf{c}) \tau(q)$, (d) $D(q)$, (e) $\alpha(q)$, (g) $f(\alpha)$ against $q$ and (f) $f(\alpha)$ against $\alpha$ 
Ray et al.

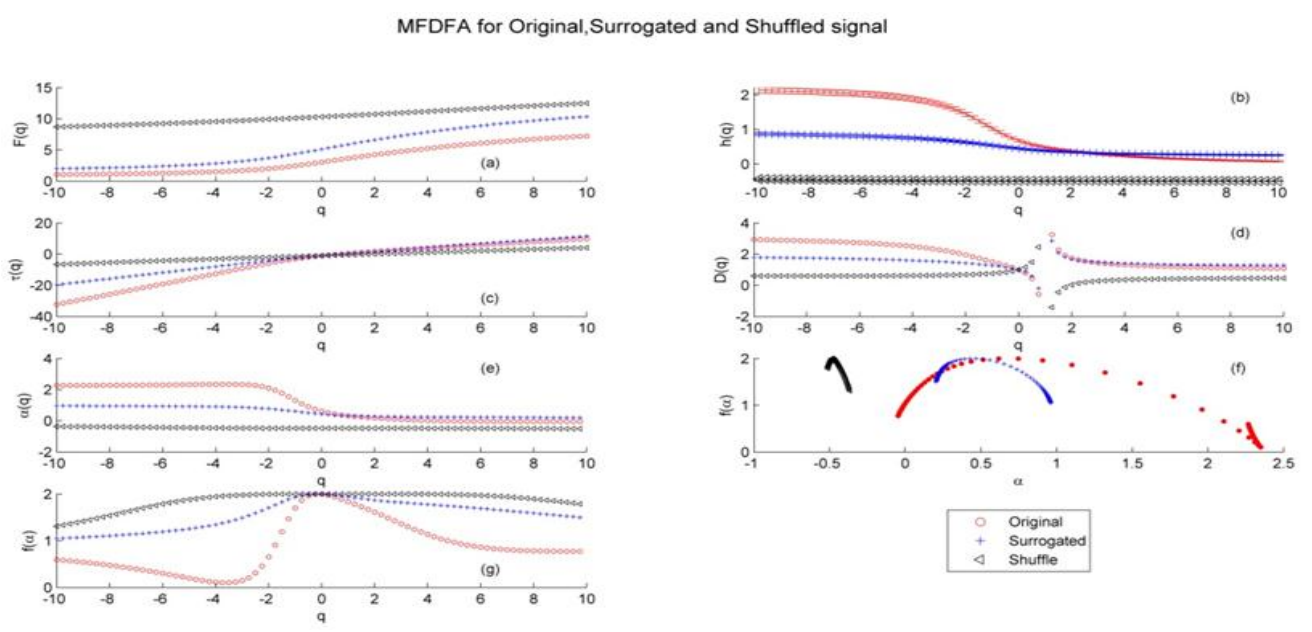

Figure 3 Comparison between original, surrogated and shuffled for Dew point Data using MFDFA3: (a) $F(q)$, (b) $h(q),(\mathbf{c}) \tau(q),(\mathbf{d}) D(q),(\mathbf{e}) \alpha(q),(\mathbf{g}) f(\alpha)$ against $q$ and (f) $f(\alpha)$ against $\alpha$
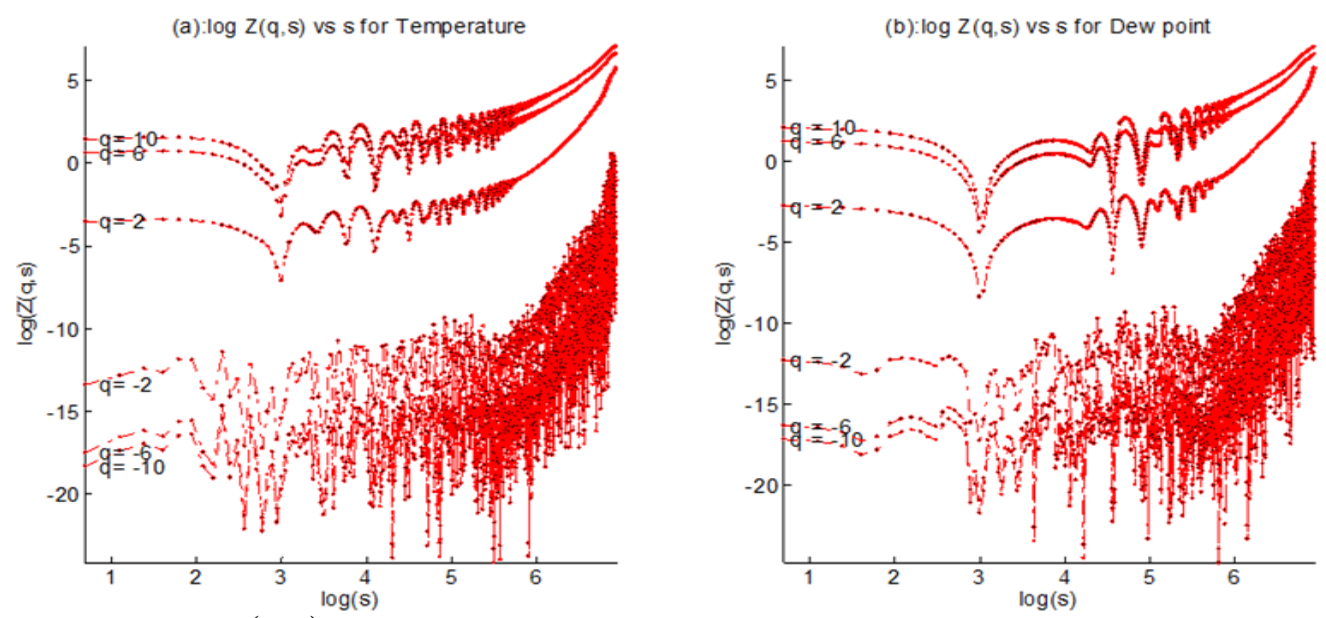

Figure $7 \log Z(q, s)$ versus $\log s$ for different $q$ using WTMM: (a) For Temperature, (b) For Dew point WTMM for Temperature and Dew point
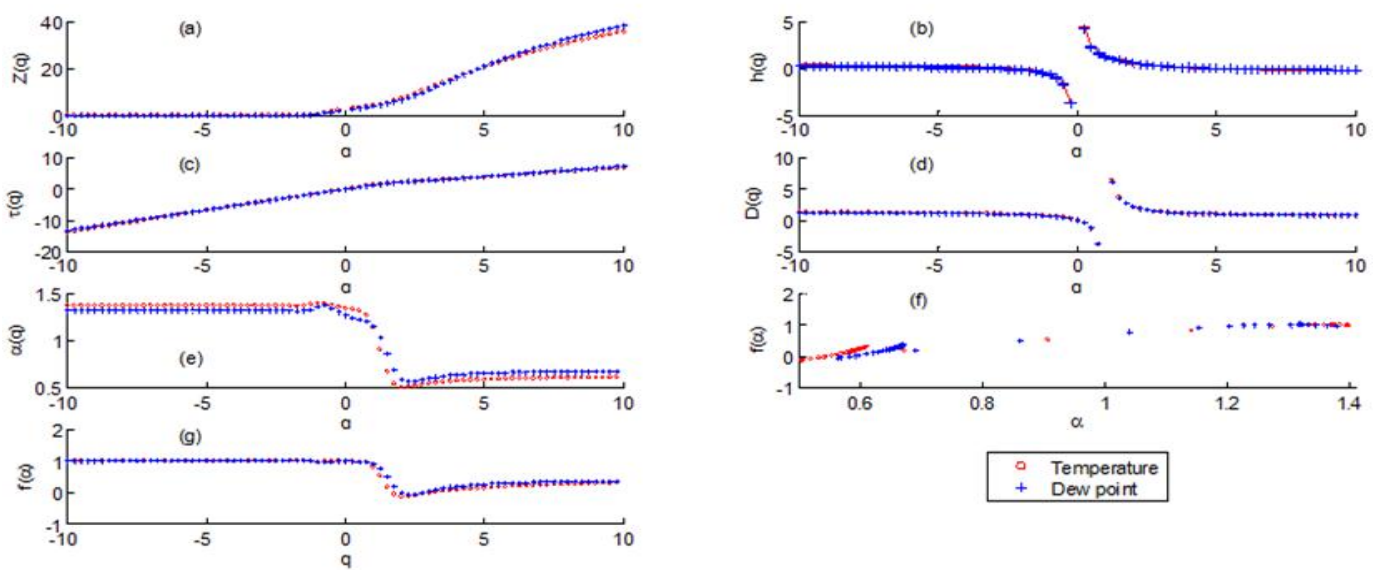

Figure 8 WTMM plots for both temperature and dew point data: (a) $Z(q),(\mathbf{b}) h(q)$, (c) $\tau(q)$, (d) $D(q)$, (e) $\alpha(q),(\mathbf{g}) f(\alpha)$ against $q$ and (f) $f(\alpha)$ against $\alpha$ 
Table 1 Quantitative results for the singularity spectrum from Figure 4, 5 and 6

\begin{tabular}{|c|c|c|c|c|c|c|}
\hline \multirow{9}{*}{ MFDFA } & \multicolumn{6}{|c|}{ Average temperature } \\
\hline & Signals & \multicolumn{2}{|c|}{$\alpha_{\min }(f=0)$} & $\alpha_{\max }(f=0)$ & $W=\alpha_{\max }-\alpha_{\min }$ & $\alpha\left(f=f_{\max }(\alpha)\right)=\alpha_{0}$ \\
\hline & Original & \multicolumn{2}{|c|}{-0.1175} & 1.4238 & 1.5413 & 0.6686 \\
\hline & Surrogate & \multicolumn{2}{|c|}{0.2180} & 1.2255 & 1.0075 & 0.6577 \\
\hline & Shuffled & \multicolumn{2}{|c|}{-0.3622} & -0.5913 & -0.2291 & -0.4791 \\
\hline & \multicolumn{6}{|c|}{ Average dew point } \\
\hline & Original & \multicolumn{2}{|c|}{-0.1314} & 2.3537 & 2.4851 & 0.7476 \\
\hline & Surrogate & \multicolumn{2}{|l|}{0.1405} & 1.0186 & 0.8781 & 0.4681 \\
\hline & Shuffled & \multicolumn{2}{|c|}{-0.3495} & -0.5303 & -0.1808 & -0.4827 \\
\hline \multirow[t]{4}{*}{ WTMM } & \multicolumn{6}{|c|}{ Average temperature } \\
\hline & \multicolumn{2}{|c|}{ Original } & 0.5153 & 1.4418 & 0.9265 & 1.3703 \\
\hline & \multicolumn{6}{|c|}{ Average dew point } \\
\hline & \multicolumn{2}{|c|}{ Original } & 0.5552 & 4.8797 & 4.3245 & 1.3167 \\
\hline
\end{tabular}

The non-linear variation and dependence of $h(q)$ and $\quad \tau(q)$ on q, as observed in Figure $5(b),(c)$ and Figure $6(b)$ and $(c)$ clearly indicates that the temperature and dew point signals have multifractal nature. From Figure $5(b)$, (c) and Figure $6(b),(c)$ it is observed that, for both the signal, fluctuation in $h(q)$ and $\tau(q)$ for the randomized data series are much flatter than those of the original signal. Figure $5(b)$ and $6(\mathrm{~b})$ show that $h(q)$ for the randomized data series which are not equal to 0.5 , symbolize that the multifractality in the temperature and dew point signals are mostly because of the broad probability density function (PDF) of the signals. This characteristics behaviour of multifractal can be highlighted the singularity spectrum as in Figure 5(f) and Figure 6(f).

The singularity spectrum width $W=\alpha_{\max }-\alpha_{\text {min }}$ reveals the range of fractal exponents and so it measures the degree of multifractality of the series. From Figure 5(f), Figure 6(f) and Table 1 it can be observe that $W$ for the signal of dew point is more than that of temperature. It signifies that the dew point signal is more sophisticated in structure as compared to that of temperature signal. Relatively strong weighted low-fractal exponents are shown by a right-skewed singularity spectrum, and relatively strong weighted high-fractal exponents are indicated by left skewed spectrum. From Figure 5(f) and Figure $6(f)$ it can be detected that the singularity spectrum is more skewed towards right side for dew point than that of temperature. Therefore we can state that the temperature signal is expected to have more high-fractal exponents than dew point signal. While using WTMM the dependence of $h(q)$ and $\tau(q)$ on $\mathrm{q}$, as seen in Figure $8(b)$ and (c), clearly signifies that both the temperature and dew point signals have multifractal behaviour which is supported by the results of MFDFA. Both WTMM and MFDFA methods agrees on the fact that the magnitude of $W$ for the signal of dew point is more than that of temperature which again reveals that the signal from dew point signal is richer in structure as related to that from temperature.

\section{Conclusion}

The analysis reveals the presence of a wide spectrum of singularities or a very sporadic multifractal structure in the present non-stationary temperature and dew point time series. It is found that for temperature and dew point, multifractality occurs because of the broadness of probability density function (PDF) which has more contribution than correlation. The temperature signal is expected to have more high-fractal exponents than dew point signal. If the range of probable fractal exponents is much wider, then the process is much 'richer' in structure. Hence the dew point signal is richer in structure as contrast to that from temperature. The singularity spectrum is more skewed towards right side for dew point than that of temperature which implies that temperature has more fine structure than dew point and the later one is having a smoothly varying appearance. The results suggest a thorough analysis of cross-correlation and self- similarity among the signals for further insight in future.

\section{Acknowledgment}

None.

\section{Conflicts of interest}

The authors have no conflicts of interest to declare. 


\section{References}

[1] Chattopadhyay N, Hulme M. Evaporation and potential evapotranspiration in India under conditions of recent and future climate change. Agricultural and Forest Meteorology. 1997; 87(1):55-73.

[2] Srivastava HN, Dewan BN, Dikshit SK, Prakash Rao GS, Singh SS, Rao KR. Decadal trends in climate over India. Mausam. 1992; 43(1):7-20.

[3] Hingane LS, Rupa Kumar K, Ramana Murty BV. Long-term trends of surface air temperature in India. International Journal of Climatology. 1985; 5(5):5218.

[4] Kumar KR, Hingane LS. Long-term variations of surface air temperature at major industrial cities of India. Climatic Change. 1988; 13(3):287-307.

[5] Rao PG. Climatic changes and trends over a major river basin in India. Climate Research. 1993; 2:21523.

[6] Kumar KR, Kumar KK, Pant GB. Diurnal asymmetry of surface temperature trends over India. Geophysical Research Letters. 1994; 21(8):677-80.

[7] Jagannathan P, Parthasarathy B. Fluctuations in the seasonal oscillations of temperature in India. Indian Journal of Meteorology and Geophysics. 1972; 23:1522.

[8] Bindu G, Anil Kumar KG. Meteorological aspects of the environment of Cochin. Doctoral Dissertation, Department of Atmospheric Sciences.1996.

[9] Ivanov PC, Amaral LA, Goldberger AL, Havlin S, Rosenblum MG, Struzik ZR, et al. Multifractality in human heartbeat dynamics. Nature. 1999:461-5.

[10] Oświęcimka P, Kwapień J, Drożdż S. Wavelet versus detrended fluctuation analysis of multifractal structures. Physical Review E. 2006; 74(1).

[11] Kantelhardt JW, Zschiegner SA, Koscielny-Bunde E, Havlin S, Bunde A, Stanley HE. Multifractal detrended fluctuation analysis of nonstationary time series. Physica A: Statistical Mechanics and its Applications. 2002; 316(1-4):87-114.

[12] Murguia JS, Perez-Terrazas JE, Rosu HC. Multifractal properties of elementary cellular automata in a discrete wavelet approach of MF-DFA. Europhysics Letters. 2009; 87(2).

[13] NOAA.Climate Data Online," NOAA, 2014. http://www7.ncdc.noaa.gov/CDO/cdosubqueryrouter.c md. Accessed 26 October 2014.

[14] Kantelhardt JW, Koscielny-Bunde E, Rego HH, Havlin S, Bunde A. Detecting long-range correlations with detrended fluctuation analysis. Physica A: Statistical Mechanics and its Applications. 2001; 295(3-4):441-54.

[15] Buldyrev SV, Goldberger AL, Havlin S, Mantegna $\mathrm{RN}$, Matsa ME, Peng CK, et al. Long-range correlation properties of coding and noncoding DNA sequences: GenBank analysis. Physical Review E. 1995; 51(5).

[16] Hajian S, Movahed MS. Multifractal detrended crosscorrelation analysis of sunspot numbers and river flow fluctuations. Physica A: Statistical Mechanics and its Applications. 2010; 389(21):4942-57.

[17] Movahed MS, Jafari GR, Ghasemi F, Rahvar S, Tabar MR. Multifractal detrended fluctuation analysis of sunspot time series. Journal of Statistical Mechanics: Theory and Experiment. 2006; 2006(02).

[18] Muzy JF, Bacry E, Arneodo A. Wavelets and multifractal formalism for singular signals: application to turbulence data. Physical Review Letters. 1991; 67(25).

[19] Hossain KM, Ghosh DN, Ghosh K. Investigating multifractality of solar irradiance data through wavelet based multifractal spectral analysis. Signal Processing: An International Journal. 2009; 3(4):83-94.

[20] Hossain KM, Ghosh DN, Ghosh K, Bhattacharya AK. Multifractality and singularity of $8 \mathrm{~B}$ solar neutrino flux signals from Sudbury Neutrino Observatory. IET Signal Processing. 2011; 5(7):690-700.

[21] Goel PK, Vidakovic B. Wavelet transformations as diversity enhancers. Institute of Statistics \& Decision Sciences, Duke University; 1995.

[22] Katul G, Vidakovic B. The partitioning of attached and detached eddy motion in the atmospheric surface layer using Lorentz wavelet filtering. Boundary-Layer Meteorology. 1996; 77(2):153-72.

[23] Stanley HE, Meakin P. Multifractal phenomena in physics and chemistry. Nature. 1988; 335(6189):4059.

[24] Daubechies I. Ten lectures on wavelets. SIAM; 1992.

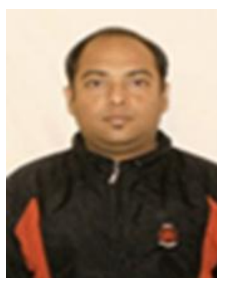

Rajdeep Ray was born on February 02, 1978. He received his B.Tech. degree in Electronics and Communication Engineering from REC, Durgapur, India, in 2002, and the M.Tech degree from National Institute of Technology, Durgapur, India, in 2008. Currently he is Assistant Professor in the Department of Electronics and Communication Engineering, Dr. B.C. Roy Engineering College, Durgapur. $\mathrm{He}$ has a total teaching experience of fourteen years. His research interest is in the area of Statistical Signal Processing, Nonlinear Dynamics and Time Series.

Email: ray.rajdeep78@gmail.com

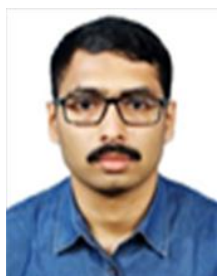

Siddhartha Dey was born in Durgapur, West Bengal, India on 29th November 1992. He received his B.Tech. degree in Electronics and Communication Engineering from Durgapur Institute of Advanced Technology and Management, Durgapur under Maulana Abul Kalam Azad University of Technology (MAKAUT), West Bengal, India, in 2015, and M.Tech. degree in Electronics and Communication Engineering (Modern Communication) from Dr. B.C. Roy Engineering College, Durgapur in 2018. 


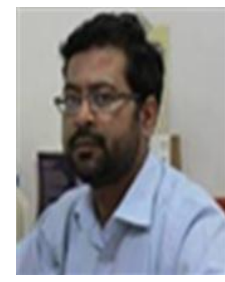

Mofazzal Hossain Khondekar was born on January 10, 1976. He received his B.Sc.(Hons.) degree in Physics and the B.Tech. degree in Instrumentation and Electronics Engineering from Jadavpur University, Kolkata, India, in 1997 and 2000, respectively, and the M.Tech. and the Ph.D. degree from National Institute of Technology, Durgapur, India, in 2008 and 2013, respectively. Currently he is Professor and HOD of the the Department of Applied Electronics and Instrumentation Engineering, Dr. B.C. Roy Engineering College, Durgapur. He has a total teaching experience of thirteen years and industry experience of five years. His research interest is in the area of Statistical Signal Processing, Nonlinear Dynamics and Time Series.

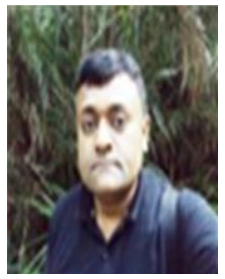

Kaushik Ghosh was born on June 25, 1974. He received his M.Sc., M.Phil., and $\mathrm{Ph} . \mathrm{D}$. degrees from the University of Calcutta, Kolkata, India, in 1998, 2000, and 2004, respectively, all in Applied Mathematics. He is currently an Assistant Professor in Mathematics with the Department of Mathematics, University of Burdwan, Burdwan, India. He has a total teaching experience of 17 years. His research interests are in the areas of Stellar and Substellar Astrophysics, Analysis of Time Series and Statistical Signal Processing, Nonlinear Systems and Dynamics, Mathematical Modeling in Biological Systems, Social and Behavioral Sciences, and Pattern Recognition. 